\title{
Article of Significant Interest Selected from This Issue by the Editors
}

\section{An RNF20-SREBP1c Axis Links Metabolism and Cell Cycle Control}

The lipogenic transcription factor sterol regulatory element-binding protein $1 \mathrm{c}$ (SREBP1c) has been associated with tumorigenesis. Although kidney cancers often exhibit ectopic lipid storage, the relationship between aberrant lipid metabolism and tumor growth is not well understood. Lee et al. (e00265-17) demonstrate that RNF20, an E3 ubiquitin ligase that targets SREBP1C, suppresses tumorigenesis by inhibiting lipogenesis and cell cycle progression in kidney cancer. The authors further show that SREBP1c regulates cell cycle progression through control of pituitary tumortransforming gene 1 (PTTG1). These findings implicate RNF20 in the regulation of SREBP1c-dependent lipogenesis in kidney cancer. 